\title{
An Interactive Data Language software package to calculate ionospheric conductivity by using numerical models
}

\section{$\operatorname{AUTHOR}(S):$}

Koyama, Yukinobu; Shinbori, Atsuki; Tanaka, Yoshimasa; Hori, Tomoaki; Nosé, Masahito; Oimatsu, Satoshi

\section{CITATION:}

Koyama, Yukinobu ... [et al]. An Interactive Data Language software package to calculate ionospheric conductivity by using numerical models. Computer Physics Communications 2014, 185(12): 3398-3405

\section{ISSUE DATE:}

2014-12

URL:

http://hdl.handle.net/2433/191212

\section{RIGHT:}

(C) 2014 Elsevier B.V.; This is not the published version. Please cite only the published version.; この論文は出版社版でありません。引用の際に は出版社版をご確認ご利用ください。 


\title{
An Interactive Data Language software package to calculate ionospheric conductivity by using numerical models
}

\author{
Yukinobu KOYAMA ${ }^{1}$ \\ ykoyama@kugi.kyoto-u.ac.jp. \\ Atsuki SHINBORI ${ }^{2}$, Yoshimasa TANAKA ${ }^{3}$, Tomoaki HORI ${ }^{4}$, Masahito \\ NOSÉ$^{1}$, Satoshi OIMATSU ${ }^{5}$
}

\begin{abstract}
The Inter-university Upper atmosphere Global Observation NETwork (IUGONET) project focuses on handling ground-based observational data of the upper atmosphere. To this end, the project members have been developing a data analysis software package which is based on Interactive Data Language (IDL). Filling the spatial gaps in observational data requires the use of numerical models. In this paper, we discuss an IDL software package for global ionospheric conductivity by integration of 3rd party numerical models. The model can be used to create further derived models.
\end{abstract}

Program Title: iug_ionospheric_cond

Catalogue identifier:

Program summary URL:

Licensing provisions: Standard CPC license, http://cpc.cs.qub.ac.uk/licence/licence.html.

Programming language: IDL-7.1.

Computer: All Ubuntu based computers. Tested on Virtual Box.

Operating system: Ubuntu 12.04 LTS.

Keywords: Ionosphere, Conductivity, Numerical Models, TDAS, UDAS, IUGONET

Classification: 1.0 .

External routines/libraries: NRLMSISE-00, IRI-2012, and IGRF-11.

Nature of problem: To calculate ionospheric conductivity.

\footnotetext{
${ }^{1}$ Data Analysis Center for Geomagnetism and Space Magnetism, Graduate School of Science, Kyoto University

${ }^{2}$ Research Institute for Sustainable Humanosphere, Kyoto University

${ }^{3}$ Space and Upper Atmospheric Science Group, National Institute of Polar Research

${ }^{4}$ Solar-Terrestrial Environment Laboratory, Nagoya University

${ }^{5}$ Solar-Planetary Electromagnetism Laboratory, Graduate School of Science, Kyoto University
} 
Solution method:

Running time:

The tests provided take only a few seconds to run.

$\mathrm{T}$

\section{Introduction}

The Inter-university Upper atmosphere Global Observation NETwork (IUGONET) [1] is a six-year research project to establish a metadata database and data analysis software to investigate the long-term variations in the upper atmosphere. Integrated links need to be created among a variety of ground-based observations made at various locations from the equator to the poles because what is observed is the result of complicated processes. The IUGONET project focuses on handling ground-based observational data of the upper atmosphere from the viewpoint of experimental science. In this paper, we treat the data from numerical models for filling the spatial gaps in observation regions.

The long-term variations in the upper and middle atmosphere have become an important area of research since the beginning of the 1990s. In the case of the ionosphere, the long-term trends in electron density, and ion composition and temperature have been associated with three possible causes: increasing greenhouse gases (Robre and Dickinson [2], Hall and Cannon [3]), long-term variation in geomagnetic activity (Danilov and Mikhailov [4], Mikhailov and Marin [5]), and secular variation in Earth's magnetic field (Foppiano et al. [6], Cnossen and Richmond [7]). Thus, long-term upper atmospheric observational data are necessary to understand the physical mechanism of the long-term variation in the upper atmosphere. However, only geomagnetic field data obtained at geomagnetic stations globally distributed in a wide region from both the poles to the equator are available for such long-term data analyses. Because the geomagnetic field variations on the ground are mainly produced by ionospheric, magnetospheric and ground-induced currents, investigation of the magnetic field variation caused by the ionospheric currents provides an important key to understanding the long-term variation in the upper atmosphere. The ionospheric current $J$ that lead to geomagnetic field variations on the ground originate from the polarization electric field $E$ and dynamo field $U \times B$ according to Ohm's law

$$
J=\Sigma \cdot(E+U \times B),
$$

where $\Sigma$ is the height-integrated ionospheric conductivity, $U$ is the neutral wind velocity, and $B$ is the geomagnetic field. The ionospheric dynamo field is strongly linked to the upper atmospheric dynamics. Therefore, to obtain information on the long-term variation in this component from geomagnetic field data analysis, we need to know the global distribution of ionospheric conductivities in the dynamo layer. Figure 1 shows a schematic picture of the height distribution of temperature, electron density, and ionospheric (Pedersen, Hall, and parallel) conductivities. The temperature and electron density structures 
in the thermosphere and ionosphere are determined by the height distributions of the neutral atmospheric density and the intensity of solar extreme ultraviolet (EUV) radiation. Because the intensity of solar EUV radiation depends on the eleven-year solar activity cycle, the temperature and electron density in these regions also exhibit an eleven-year periodic variation. The ionospheric conductivities determined by the atmospheric conditions in the thermosphere and ionosphere exhibits a similar variation with an eleven-year period. Therefore, by removing solar activity dependence of ionospheric currents estimated from geomagnetic field data on the ground using the long-term calculated ionospheric conductivities, we can investigate the effect of lower atmospheric disturbances on the long-term variation in the upper atmosphere. A web service providing $\Sigma$ calculations for arbitrary location, time, and date has been released to the public [8]. In principle, using this service one should be able to calculate the long-term variation of $\Sigma$ at an arbitrary site and a world map of $\Sigma$ for an arbitrary height. However, calculating them with sufficient resolution is not practical because the site limits the band of the network. Moreover, the site describes only an overview of the calculation. Thus, it is impossible to verify the validity of the calculation results because the implementations are not available. To solve these problems, we implemented a library to calculate $\Sigma$ and opened all source codes including unit test codes to the public on GitHub [9].

\section{Equations}

We next present the two kinds of ionospheric conductivity and collision frequency equations that we implemented.

\subsection{Maeda's Ionospheric Conductivity Equations and Banks's Collision fre- quency Equations}

The Ionospheric conductivities are defined by Maeda [10] as

$$
\left\{\begin{aligned}
\sigma_{0} & =\frac{\mathrm{e}^{2} N_{e}}{m_{e} \nu_{e}} \\
\sigma_{1} & =\frac{(1+\kappa) \nu_{e}^{2}}{(1+\kappa)^{2} \nu_{e}^{2}+\omega_{e}^{2}} \sigma_{0}, \\
\sigma_{2} & =\frac{\omega_{e} \nu_{e}}{(1+\kappa)^{2} \nu_{e}^{2}+\omega_{e}^{2}} \sigma_{0},
\end{aligned}\right.
$$

where $\sigma_{0}, \sigma_{1}$, and $\sigma_{2}$ are the parallel, Pedersen, and Hall conductivity, respectively, e is the elementary electric charge, $N_{e}$ is the electron density, $m_{e}$ is the mass of electron, $\nu_{e}$ is the average collision frequency of electrons, and $\omega_{e}$ is the average cyclotron frequency of electrons. Here, $\kappa$ is defined as

$$
\kappa=\frac{\omega_{e} \omega_{i}}{\nu_{e} \nu_{i}}
$$

where $\omega_{i}$ is the average cyclotron frequency of ions, and $\nu_{i}$ is the average collision frequency of ions. The collision frequencies are defined by Banks and Kocharts 
[11] as

$$
\left\{\begin{array}{l}
\nu_{e}=\nu_{e n}+\nu_{e i}, \\
\nu_{i}=\nu_{i n}
\end{array}\right.
$$

where $\nu_{e n}$ is the average collision frequency between electrons and neutral particles, $\nu_{e i}$ is the average collision frequency between electrons and ions, and $\nu_{i n}$ is the average collision frequency between ions and neutral particles. The cyclotron frequencies are defined as

$$
\left\{\begin{array}{l}
\omega_{e}=\frac{\mathrm{e} B}{m_{e}}, \\
\omega_{i}=\frac{\mathrm{e} B}{m_{i}}
\end{array}\right.
$$

where $B$ is the total magnetic flux, and $m_{i}$ is the mass of the ions. The various collision frequencies are defined as

$$
\left\{\begin{array}{l}
\nu_{e n}=\nu_{e n}\left(\mathrm{~N}_{2}\right)+\nu_{e n}\left(\mathrm{O}_{2}\right)+\nu_{e n}(\mathrm{O})+\nu_{e n}(\mathrm{H})+\nu_{e n}\left(\mathrm{H}_{e}\right) \\
\nu_{e n}\left(\mathrm{~N}_{2}\right)=2.33 \times 10^{-11} n\left(\mathrm{~N}_{2}\right)\left[1-1.2 \times 10^{-4} T_{e}\right] T_{e} \\
\nu_{e n}\left(\mathrm{O}_{2}\right)=1.8 \times 10^{-10} n\left(\mathrm{O}_{2}\right)\left[1+3.6 \times 10^{-2} T_{e}^{1 / 2}\right] T_{e}^{1 / 2} \\
\nu_{e n}(\mathrm{O})=8.2 \times 10^{-10} n(\mathrm{O}) T_{e}^{1 / 2} \\
\nu_{e n}(\mathrm{H})=4.5 \times 10^{-9} n(\mathrm{H})\left[1-1.35 \times 10^{-4} T_{e}\right] T_{e}^{1 / 2} \\
\nu_{e n}(\mathrm{He})=4.6 \times 10^{-10} n(\mathrm{He}) T_{e}^{1 / 2}
\end{array}\right.
$$

where $\nu_{e n}\left(\mathrm{~N}_{2}\right), \nu_{e n}\left(\mathrm{O}_{2}\right), \nu_{e n}(\mathrm{O}), \nu_{e n}(\mathrm{H})$, and $\nu_{e n}\left(\mathrm{H}_{\mathrm{e}}\right)$ are average collision frequencies between electrons and $\mathrm{N}_{2}, \mathrm{O}_{2}, \mathrm{O}, \mathrm{H}$, and $\mathrm{H}_{\mathrm{e}}$, respectivily. The numbers of $\mathrm{N}_{2}, \mathrm{O}_{2}, \mathrm{O}, \mathrm{H}$, and $\mathrm{H}_{\mathrm{e}}$ are $n\left(\mathrm{~N}_{2}\right), n\left(\mathrm{O}_{2}\right), n(\mathrm{O}), n(\mathrm{H})$, and $n\left(\mathrm{H}_{\mathrm{e}}\right)$, respectivily. Here $T_{e}$ is the average electron temperature. The collision frequency $\nu_{e i}$ is defined by as

$$
\nu_{e i}=\frac{4(2 \pi)^{1 / 2}}{3} \frac{Z_{i}^{2} \mathrm{e}^{4} \ln \Lambda}{m_{e}^{1 / 2}\left(k T_{e}\right)^{3 / 2}} n_{i},
$$

where $Z_{i}$ is the atomic number, $\ln \Lambda$ is the Coulomb logarithm, $k$ is the Boltzmann's constant, and $n_{i}$ is the number of ions. The plasma parameter $\Lambda$ is defined as

$$
\Lambda=\frac{Z_{1} Z_{2} \mathrm{e}^{2}}{\mu g \lambda_{D}},
$$

where $Z_{1}$ and $Z_{2}$ are two charge states, $\mu$ is the reduced two-particle mass, and $g$ is their relative velocity. The Debye length $\lambda_{D}$ is defined as

$$
\lambda_{D}=6.9 \sqrt{\frac{T_{e}}{n_{e}}},
$$

where $n_{e}$ is the number of electrons. The collision frequency $\nu_{i n}$ is defined as

$$
\nu_{i n}=2.6 \times 10^{-9} n_{n}\left(\frac{\alpha_{0}}{\mu_{A}}\right)^{1 / 2}
$$


where $\alpha_{0}$ is the atomic polarizability, $\mu_{A}$ is the reduced mass, and $n_{n}$ is the number of neutral particles. The polarizabilities of neutral gases are listed in Table 1.

\subsection{Richmond's Ionospheric Conductivity Equations and Collision Frequency Equations}

Another set of ionospheric conductivity equations are defined as

$$
\left\{\begin{aligned}
\sigma_{0} & =\frac{\mathrm{e}^{2} N_{e}}{m_{e}\left(\nu_{e n \|}+\nu_{e i \|}\right)}, \\
\sigma_{1} & =\frac{N_{e} \mathrm{e}}{B}\left(\frac{\nu_{i n} \omega_{i}}{\nu_{i n}^{2}+\omega_{i}^{2}}+\frac{\nu_{e n \perp} \omega_{e}}{\nu_{e n \perp}^{2}+\omega_{e}^{2}}\right), \\
\sigma_{2} & =\frac{N_{e} \mathrm{e}}{B}\left(\frac{\omega_{e}^{2}}{\nu_{e n \perp}^{2}+\omega_{e}^{2}}+\frac{\omega_{i}^{2}}{\nu_{i n}^{2}+\omega_{i}^{2}}\right),
\end{aligned}\right.
$$

where $\nu_{e n *}$ is the average collision frequency between electrons and neutral particles, and $\nu_{e i *}$ is the average collision frequency between electrons and ions by Volland [13]. Here the subscript $\|$ signifies the component parallel to $B$, and $\perp$ signifies the component perpendicular to $B$. The collision frequencies are defined as

$$
\left\{\begin{array}{l}
\nu_{e n \|}=\left[4.6 n\left(\mathrm{~N}_{2}\right) R_{e}^{0.95}+4.3 n\left(\mathrm{O}_{2}\right) R_{e}^{0.79}+1.5 n(\mathrm{O}) R_{e}^{0.85}\right] \times 10^{-15} \\
\nu_{e i \|}=\left(1.84 \times 10^{-6}\right)(\ln \Lambda) N_{e} T_{e}^{-3 / 2} \\
\nu_{e \|}=\nu_{e n \|}+\nu_{e i \|}
\end{array}\right.
$$

where

$$
R_{e}=\frac{T_{e}}{300} .
$$

The Coulomb logarithm $\ln \Lambda$ is defined here as

$$
\ln \Lambda=16.33+0.5 \ln \frac{T_{e}^{3}}{N_{e}} .
$$

The collision frequencies are defined as

$$
\left\{\begin{aligned}
\nu_{i n} & =\nu_{i n}\left(\mathrm{NO}^{+}\right)+\nu_{i n}\left(\mathrm{O}_{2}^{+}\right)+\nu_{i n}\left(\mathrm{O}^{+}\right) \\
\nu_{i n}\left(\mathrm{NO}^{+}\right)= & {\left[3.4 n\left(\mathrm{~N}_{2}\right) R_{i}^{-0.16}+3.4 n\left(\mathrm{O}_{2}\right) R_{i}^{-0.16}+1.9 n(\mathrm{O}) R_{i}^{-0.19}\right] } \\
& \times 10^{-16}, \\
\nu_{i n}\left(\mathrm{O}_{2}^{+}\right)= & {\left[3.3 n\left(\mathrm{~N}_{2}\right) R_{i}^{-0.17}+6.1 n\left(\mathrm{O}_{2}\right) R_{i}^{0.37}+1.8 n(\mathrm{O}) R_{i}^{-0.19}\right] } \\
& \times 10^{-16}, \\
\nu_{i n}\left(\mathrm{O}^{+}\right)= & \left.5.4 n\left(\mathrm{~N}_{2}\right) R_{i}^{-0.20}+7.0 n\left(\mathrm{O}_{2}\right) R_{i}^{0.05}+8.9 n(\mathrm{O}) R_{i}^{0.5}\right] \\
& \times 10^{-16}, \\
\nu_{e n \perp} & {\left[7.2 n\left(\mathrm{~N}_{2}\right) R_{e}^{0.95}+5.2 n\left(\mathrm{O}_{2}\right) R_{e}^{0.79}+1.9 n(\mathrm{O}) R_{e}^{0.85}\right] } \\
& \times 10^{-15},
\end{aligned}\right.
$$


where $\nu_{i n}\left(\mathrm{NO}^{+}\right), \nu_{i n}\left(\mathrm{O}_{2}^{+}\right)$, and $\nu_{i n}\left(\mathrm{O}^{+}\right)$are the average collision frequencies between neutral particles and $\mathrm{NO}^{+}, \mathrm{O}_{2}^{+}$, and $\mathrm{O}^{+}$, respectively. $R_{i}$ is defined as

$$
R_{i}=\frac{T_{n}+T_{i}}{1000}
$$

where $T_{n}$ is the average temperature of neutral particles and $T_{i}$ is the average temperature of ions.

\subsection{Common Equations}

The current densities are defined as

$$
\left\{\begin{array}{l}
j_{x}=\sigma_{x x} E_{x}+\sigma_{x y} E_{y}, \\
j_{y}=-\sigma_{x y} E_{x}+\sigma_{y y} E_{y},
\end{array}\right.
$$

where $E_{x}$ and $E_{y}$ are, respectively, the $x$ and $y$ components of the horizontal electric field. We took the coordinates in which $x$ is the magnetic north and $y$ is the magnetic east. The two-dimensional ionospheric conductivities $\sigma_{x x}, \sigma_{y y}$, and $\sigma_{x y}$ are defined as

$$
\left\{\begin{aligned}
\sigma_{x x} & =\frac{\sigma_{0} \sigma_{1}}{\sigma_{1} \cos ^{2} I+\sigma_{0} \sin ^{2} I} \\
\sigma_{y y} & =\frac{\sigma_{0} \sigma_{1} \sin ^{2} I+\left(\sigma_{1}^{2}+\sigma_{2}^{2}\right) \cos ^{2} I}{\sigma_{1} \cos ^{2} I+\sigma_{0} \sin ^{2} I} \\
\sigma_{x y} & =\frac{\sigma_{0} \sigma_{2} \sin I}{\sigma_{1} \cos ^{2} I+\sigma_{0} \sin ^{2} I}
\end{aligned}\right.
$$

where $I$ is the geomagnetic dip angle. Their height-integrated values of them are $\Sigma_{x x}, \Sigma_{y y}$, and $\Sigma_{x y}$.

\section{Programs}

\subsection{IDL, TDAS, UDAS, and the Ionospheric Conductivity Model}

We show the dependency diagram of IDL, TDAS, UDAS, and the ionospheric conductivity model in Figure 2. We adopted the commercially available Interactive Data Language (IDL) as the base software [14]. The Themis Data Analysis Software (TDAS) suite is written in IDL, which is widely used in fields of solar-terrestrial physics, especially for handling satellite data [15]. The package of TDAS contains the procedures which deal with satellite, such as THEMIS, ACE, WIND, and GOES. The iUgonet Data Analysis Software (UDAS) is based on TDAS for handling the ground-based observational data [16]. The package of UDAS contains the procedures which deal with geomagnetic data, image data by all sky imager for aurora, image data by solar telescope, wind direction and velocity data by radar, and so on. The IDL is commercial software but TDAS and UDAS are distributed freely. Users can obtain data without regard to data location because each load procedure accesses data through the network, and then the data are automatically downloaded onto the users' computer. Users 
can analyze the data without any concerns about data format because an intermediate variable (called "TPlot") absorbs the difference in data formats for time series data. The basic procedures for data analysis are prepared by TDAS. The TDAS and UDAS framework is good at handling time series data but it is poor at handling two-dimensional map data such as an ionospheric conductivity model. Therefore, the ionospheric conductivity model is not based on TDAS and UDAS; rather, it is based on IDL. It is effective to use them because it becomes possible to compare ground-based observational data, satellite data, and data obtained by using the ionospheric conductivity model in arbitrary time. The ionospheric conductivity model is also freely distributed via GitHub [9]. In addition, the ionospheric conductivity model treats variables with single precision.

\subsection{Numerical Models That Constitute the Ionospheric Conductivity Model}

The ionospheric conductivity model implemented using the equations in Section 2 needs some input parameters. Three models are required. These are detailed in the following. The International Reference Ionosphere 2012 (IRI-2012) is an empirical standard model of the ionosphere. For a given location, time, and date, it provides monthly averages of the electron density, electron temperature, ion temperature, and ion composition in the altitude range from 50 to $2000 \mathrm{~km}$ [17][18]. NRLMSISE-00 is an empirical, global model of the Earth's atmosphere from ground to space. It models the temperatures and densities of the atmosphere's components. NRL and MSIS stand for the U.S. Naval Research Laboratory and Mass Spectrometer and Incoherent Scatter radar respectively. Two primary data sources were used for development of earlier versions of the model. E indicates that the model extends from the ground through the exosphere and 00 is the year of release (2000) [19]. The 11th Generation International Geomagnetic Reference Field model (IGRF-11) model is used to calculate Earth's main magnetic field and is used widely in studies of the Earth's deep interior, its crust and its ionosphere and magnetosphere [20][21].

All models called by the ionospheric conductivity model call were written in Fortran. Then, we build executable files. The ionospheric conductivity model calls them by using a system call and file input and output (I/O) because some of them demand the interactive parameter input. The calculation results are retrieved via a file. It is good not to modify the original models in order to prevent mixing of errors. However, the interface is poor from the viewpoint of interoperability and file I/O overhead. The model reused, which is written in an object-oriented programming language, has more desirable interoperability.

\subsection{Internal Database as Cache}

The large number of nesting loops for time and location led to long computation times for calculating world maps from the ionospheric conductivity model. In the original TDAS framework, the observational data are downloaded from the network and managed as a local file. To use the file again, the local file is used without the need to download it from the network. Such a cache mechanism 
is also effective in model calculations. We implemented this cache mechanism in the model calculations by using the free Relational DataBase Management System (RDBMS) SQLite [22]. The database managed by SQLite can be accessed from any arbitrary software that can use Open Database Connectivity (ODBC) or Java Database Connectivity (JDBC). We show the sequence diagram of the ionospheric conductivity model in Figure 3. Once a result is calculated, it is saved in the cache database.

\subsection{Unit Test}

To verify the validity of each part of the model, numerous unit test codes were prepared. Thus, the model does not need to be treated as a black box, and users can verify it by themselves. An example that calls one unit test is the following:

IDL> iug_load_ionospheric_cond_diagnostics_1_00

\subsection{Installation}

All processes in this subsection were verified on Ubuntu 12.04 LTS (64 bit) with an ifort 12.0.2 20110112 environment. As shown in Figure 2, the ionospheric conductivity model depends on IDL. Moreover, to substitute the calculated ionospheric conductivity for "TPlot" on the TDAS and UDAS framework and treat it as time series data, TDAS and UDAS need to be installed in series. Hence, prior to the installation of the ionospheric conductivity model, the user has to install IDL. This section focuses on describe the installation of the ionospheric conductivity model. After installation of IDL, the user downloads the ionospheric conductivity model package from GitHub [9]. The ionospheric conductivity model package includes build.xml for Ant to set up the ionospheric conductivity model. Before executing the Ant process, the user has to specify $\$\{F C\}$ for the Fortran compiler and $\$\{$ UDAS_EXTRA $\}$ for the installation folder in build.properties. Then the user executes "ant install" as the root user from the command line, which installs all programs onto the user's computer. The user bahind proxy server has to specify the proxy server for Ant.

\section{Examples}

We show some results calculated using the ionospheric conductivity model. Figure 4 shows the height profile of ionospheric conductivities $\sigma_{0}, \sigma_{1}$, and $\sigma_{2}$ calculated by using the equations in Section 2.1 in the altitude range from 100 to $400 \mathrm{~km}$ at 12:00 universal time (UT) on January 1, 2000. Figure 5, 6, and 7 show the world map of ionospheric conductivities $\sigma_{0}, \sigma_{1}$, and $\sigma_{2}$ calculated by the equations in Section 2.1 at an altitude of $100 \mathrm{~km}$ at 12:00 UT on January 1, 2000 , respectively. When repeating such calculations using various parameters, using the internal database is effective. The ionospheric conductivities $\sigma_{x x}, \sigma_{y y}$, and $\sigma_{x y}$ and the height-integrated ionospheric conductivities $\Sigma_{0}, \Sigma_{1}$, and $\Sigma_{2}$ of arbitrary height ranges are also calculable. Figure 5, 6, and 7 are obtained by imprelemting the following command: 
IDL> iug_load_ionospheric_cond_map, yуyy=2000, mmdd=101,

ltut=1, time=12, height_bottom=100, height_top=100,

height_step=0, algorithm=1, reso_lat=5, reso_lon=5

Here "algorithm=1" means that the equations in Section 2.1 are used. We used a $5^{\circ}$ mesh for both latitude and longitude for these examples. For actual research use, a $1^{\circ}$ mesh will be needed around the dip equator.

Next, we show a stereo plot of the height-integrated ionospheric conductivity $\Sigma_{0}$ calculated by using the equations in Section 2.1 in the altitude range from 100 to $120 \mathrm{~km}$ at 12:00 UT on January 1, 2000. We show the time dependency of the height profile of the ionospheric conductivity $\sigma_{0}$ calculated by using the equations in Section 2.1 in the altitude range from 100 to $300 \mathrm{~km}$.

\section{Reuse of the Model}

Figure 10 shows the dependency diagram of the models. The ionospheric conductivity model was created by NRLMSISE-00, IRI-2012, and IGRF-11 models. The IRI-2012 and IGRF-11 models can be reused by creating an Alfvén conductivity model. The Alfvén conductivity is defined as

$$
\Sigma_{A}=\frac{1}{\mu_{0} v_{A}}
$$

where $\mu_{0}$ is the absolute permeability of vacuum and $v_{A}$ is the Alfvén velocity at the electron density maximum altitude [24]. The dispersion relation is given by

$$
\omega=k v_{A}
$$

where $\omega$ is the angular frequency and $k$ is the wave number vector in the direction of the external magnetic field. The propagation velocity is given by

$$
v=\frac{\omega}{k}=\frac{B}{\sqrt{\mu_{0} \rho}},
$$

where $\rho$ is the average ion mass.

The Pedersen conductivity obtained from the ionospheric conductivity model and the Alfvén conductivity are also reused for derivation of the ionospheric reflectance of Alfvén waves. The reflectance of Alfvén wave is given by

$$
R=\frac{\Sigma_{A}-\Sigma_{P}}{\Sigma_{A}+\Sigma_{P}},
$$

where $\Sigma_{P}$ is the height-integrated Pedersen conductivity. We also implemented these models as one of our case study examples of ionospheric conductivity model reuse [25]. 


\section{Conclusions}

We implemented the IDL software package to calculate ionospheric conductivity by using numerical models. To show the interoperability of the model, we also implemented an Alfvén conductivity model as one of our case study examples of mashup with the ionospheric conductivity model. We have opened all source codes including unit test codes to the public on GitHub. This model implementation is expected to become the standard platform for discussing ionospheric conductivity.

\section{Acknowledgment}

This research is supported by the joint research of the Solar-Terrestrial Environment Laboratory, Nagoya University. The IUGONET project is supported by the Special Educational Research Budget (Research Promotion) [FY2009] and the Special Budget (Project) [FY2010 and later years] from the Ministry of Education, Culture, Sports, Science and Technology (MEXT), Japan.

Thanks are owed to Dr. J. Michael Picone (Naval Research Laboratory) for providing the NRLMSISE-00 atmospheric density model, to Dr. D. Bilitza (NASA) for providing the IRI-2012 ionospheric model, and to IAGA Working Group V-MOD for providing the program for synthesizing geomagnetic field values from IGRF-11.

\section{References}

[1] http://www.iugonet.org/en/.

[2] Robre, R. G., and R. E. Dickinson. How will changes in carbon dioxide and methane modify the mean structure of the mesosphere and thermosphere? Geophys. Res. Lett., 16, 1441-1444, 1989.

[3] Hall, C. M., and P. S. Cannon. Trends in foF 2 above Troms $\varnothing\left(69^{\circ} \mathrm{N} 19^{\circ} \mathrm{E}\right)$. DOI: $10.1029 / 2002$ GL016259.

[4] Danilov, A. D., and A. V. Mikhailov. Spatial and seasonal variations of the foF2 long-term trends. Ann. Geophys., 17 1239, 1999.

[5] Mikhailov, A. V., and D. Marin. Geomagnetic control of the foF2 long-term trends. Ann. Geophys. 18, 2000.

[6] Foppiano, A. J., L. Cid, and V. Jara. Ionospheric long-term trends for South American mid-latitudes. J. Atmos. Sol.-Terr. Phys., 61, 717-723, 1999.

[7] Cnossen, I., and A. D. Richmond. Modelling the effects of changes in the Earth's magnetic field from 1957 to 1997 on the ionospheric hmF2 and foF2 parameters. J. Atmos. Sol.-Terr. Phys., 70, 1512-1524, 2008.

[8] http://wdc.kugi.kyoto-u.ac.jp/ionocond/sigcal/index.html. 
[9] https://github.com/iugonet/IonosphericConductivity/.

[10] Maeda, K. Conductivity and drift in the ionosphere. J. Atmos. Terr. Phys., 39, 1041-1053, 1977.

[11] Banks, P. M., and G. Kocharts. Aeronomy Part A. Academic, New York, 1973.

[12] Ashera R. L., D. Bellerta, T. Buthelezia, V. Lewisa, and P.J. Brucata. A measure of the effective electric-dipole polarizability of argon. Chem. Phys. Lett., 234, 113, 1995.

[13] Volland, H. Handbook of Atmospheric Electrodynamics, Volume II, CRC, Boca Raton, 1995.

[14] http://www.exelisvis.com/.

[15] http://themis.ssl.berkeley.edu/software.shtml.

[16] http://www.iugonet.org/en/software.html.

[17] Bilitza D., and B. Reinisch. International Reference Ionosphere 2007: Improvements and new parameters. Adv. Space Res. 42(4), 599-609, 2008.

[18] http://iri.gsfc.nasa.gov/.

[19] Picone, J. M., A. E. Hedin, D. P. Drob, and A. C. Aikin. NRLMSISE-00 empirical model of the atmosphere: Statistical comparisons and scientific issues. J. Geophys. Res., 107(A12), 1468, 2002.

[20] Finlay, C. C., S. Maus, C. D. Beggan, T. N. Bondar, A. Chambodut, T. A. Chernova, A. Chulliat, V. P. Golovkov, B. Hamilton, M. Hamoudi, R. Holme, G. Hulot, W. Kuang, B. Langlais, V. Lesur, F. J. Lowes, H. Lühr, S. Macmillan, M. Mandea, S. McLean, C. Manoj, M. Menvielle, I. Michaelis, N. Olsen, J. Rauberg, M. Rother, T. J. Sabaka, A. Tangborn, L. Tøffner-Clausen, E. Thébault, A. W. P. Thomson, I. Wardinski, Z. Wei, and T. I. Zvereva. International Geomagnetic Reference Field: The Eleventh Generation. Geophys. J. Int., 183, 1216-1230, 2010.

[21] http://www.ngdc.noaa.gov/IAGA/vmod/igrf.html.

[22] http://www.sqlite.org/.

[23] http://earth.google.com/.

[24] Belyaev, P. P., S. V. Polyakov, V. O. Rapoport, and V. Yu Trakhtengerts. The ionospheric Alfvén resonator. J. Atmos. Terr. Phys., 52, 781-788, 1990.

[25] Oimatsu, S. Ionospheric Alfvén resonance observed at a low-latitude station, Wakura. Graduate School of Science, Bachelor Thesis, Kyoto University, 2012. 


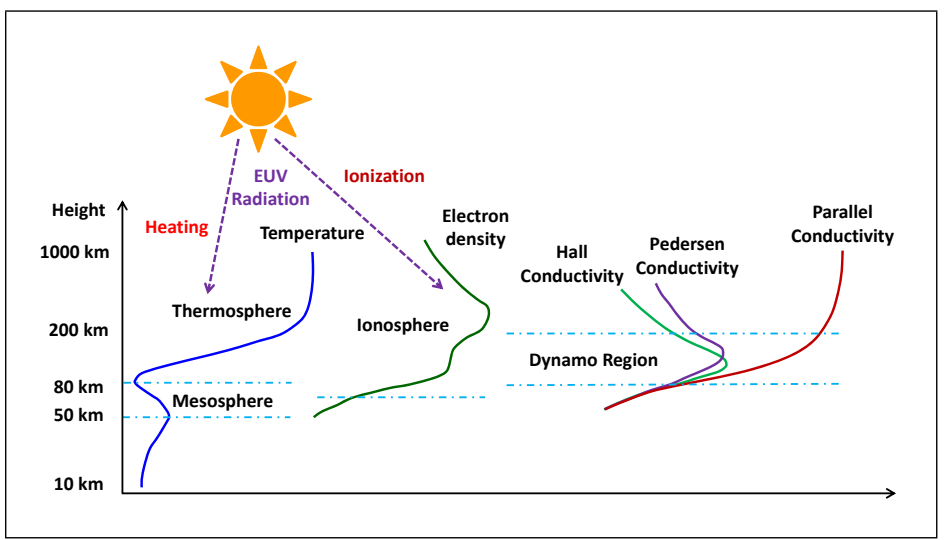

Figure 1: Relationship between solar activity and upper atmospheric variation.

Table 1: Polarizabilities of neutral gases (from Banks and Kocharts [11] and Ashera et al. *[12]).

\begin{tabular}{ll}
\hline Neutral gas & $\alpha_{0}\left(10^{-24} \mathrm{~cm}^{3}\right)$ \\
\hline $\mathrm{N}_{2}$ & 1.76 \\
$\mathrm{O}_{2}$ & 1.59 \\
$\mathrm{O}$ & $0.79 \pm 0.02$ \\
$\mathrm{H}$ & 0.667 \\
$\mathrm{He}$ & $0.21 \pm 0.01$ \\
$\mathrm{~N}$ & $1.1 \pm 0.1$ \\
$\mathrm{NH}_{3}$ & 2.22 \\
$\mathrm{Ar}$ & $1.49^{*}$ \\
\hline
\end{tabular}

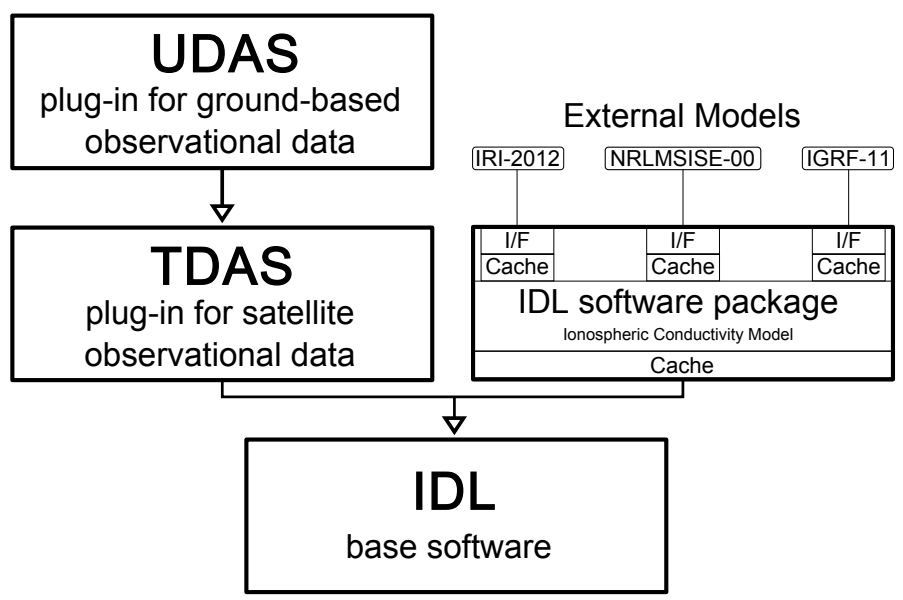

Figure 2: Dependency diagram of IDL, TDAS, UDAS, and the ionospheric conductivity model. 


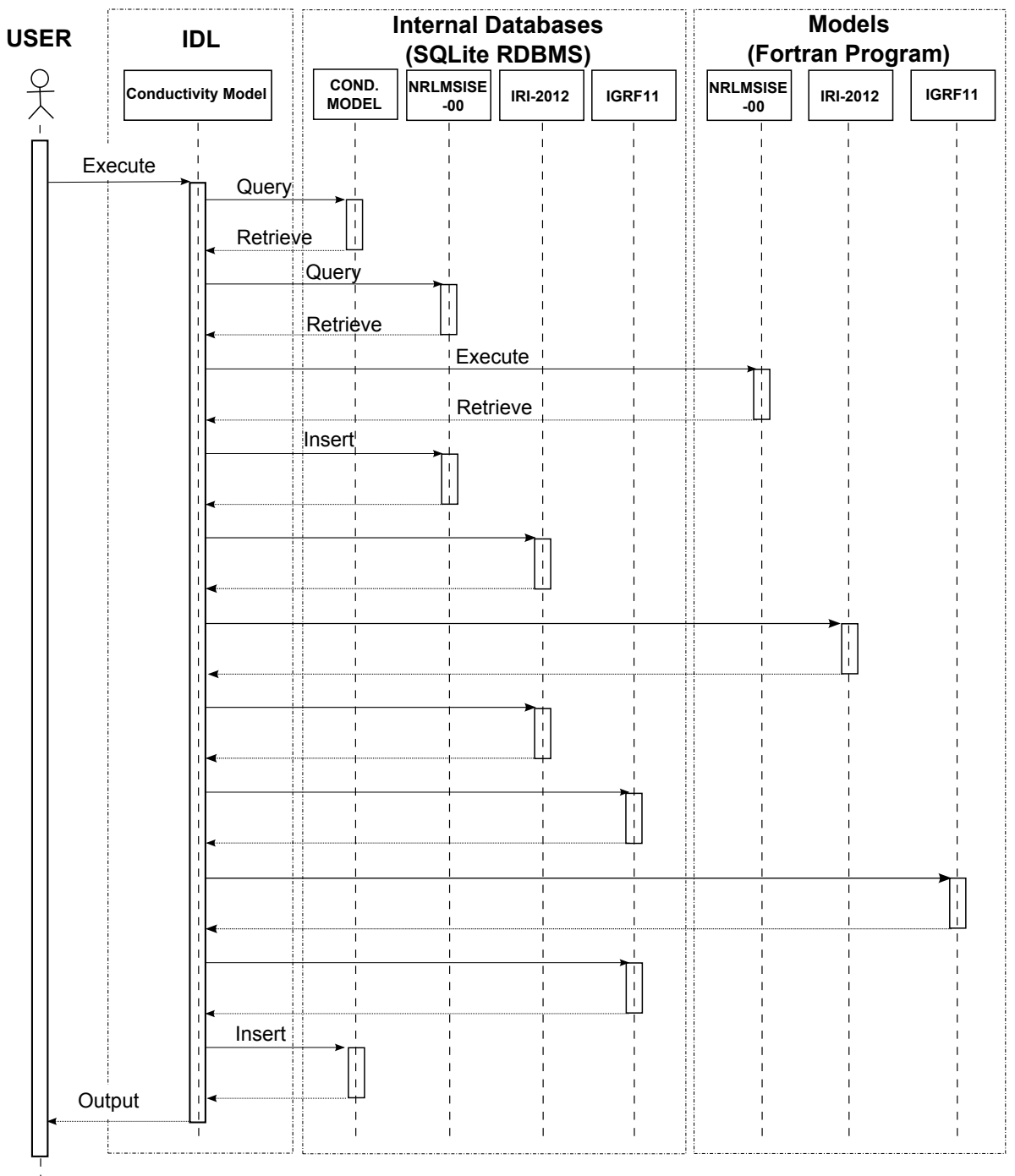

Figure 3: Sequence diagram of the ionospheric conductivity model. When ionospheric conductivity model is executed on IDL, the cache on RDBMS is accessed first. If the same parameters were calculated in the past, the calculation results will be returned immediately from the cache. When the parameters which were not calculated in the past is given, a calculation results are inserted to the cache after model calculation is executed. In addition, it is the same about three models which constitute ionospheric conductivity model. 


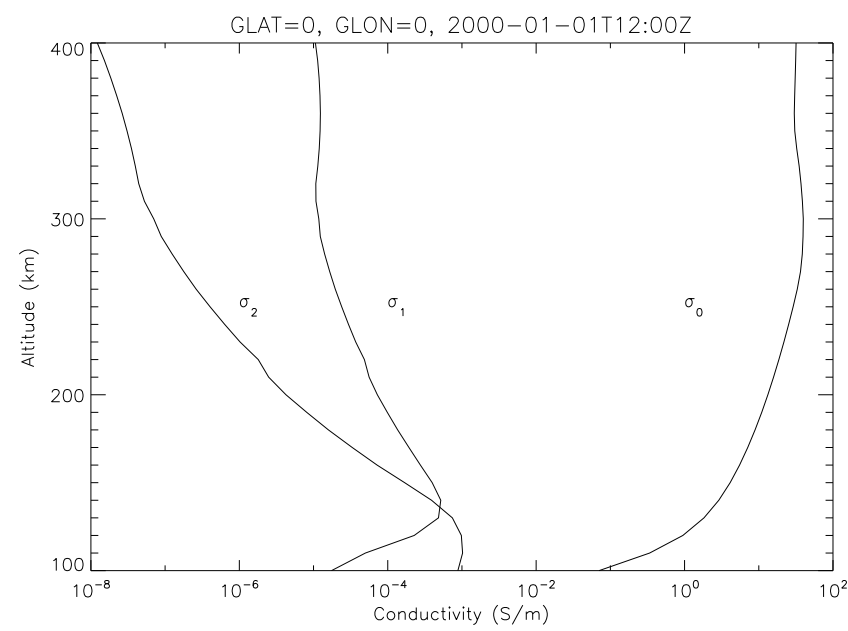

Figure 4: Heght profile of the ionospheric conductivity $\sigma_{0}, \sigma_{1}$, and $\sigma_{2}$ calculated by using the equations in Section 2.1 in the altitude range from 100 to $400 \mathrm{~km}$ at 12:00 UT on January 1, 2000

2000-01-01T12:00Z, $0100 \mathrm{~km}$

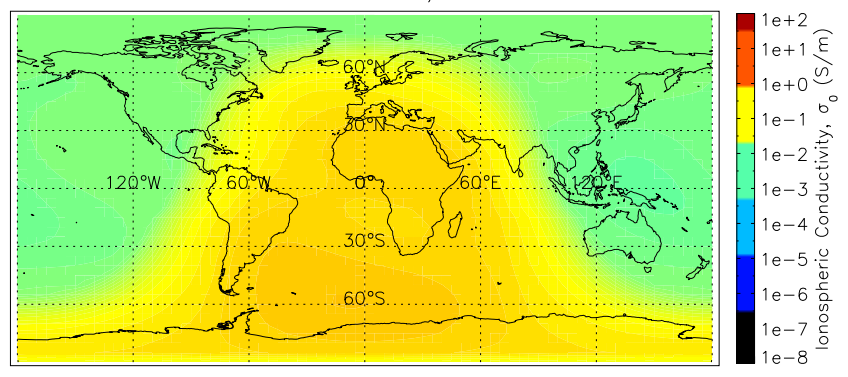

Figure 5: World map of ionospheric conductivity $\sigma_{0}$ calculated by using the equations in Section 2.1 at an altitude $100 \mathrm{~km}$ at 12:00 UT on January 1, 2000. 


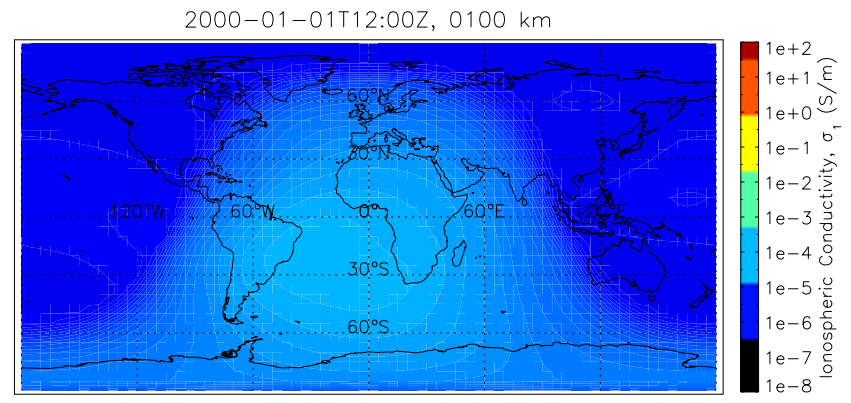

Figure 6: World map of ionospheric conductivity $\sigma_{1}$ calculated by using the equations in Section 2.1 at an altitude $100 \mathrm{~km}$ at 12:00 UT on January 1, 2000.

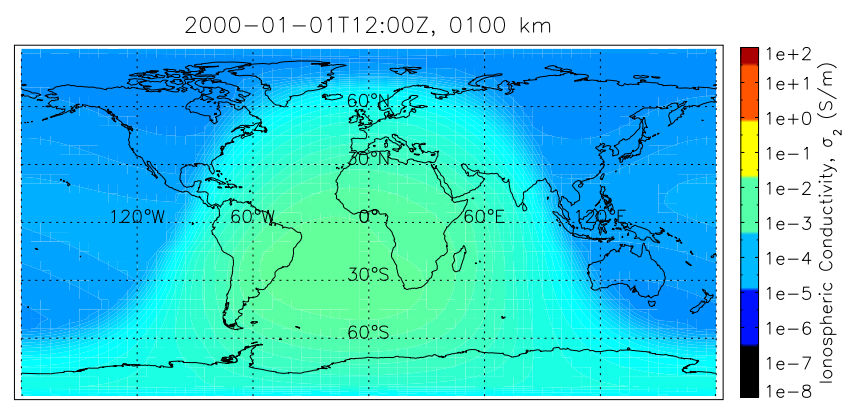

Figure 7: World map of ionospheric conductivity $\sigma_{2}$ calculated by using the equations in Section 2.1 at an altitude $100 \mathrm{~km}$ at 12:00 UT on January 1, 2000. 


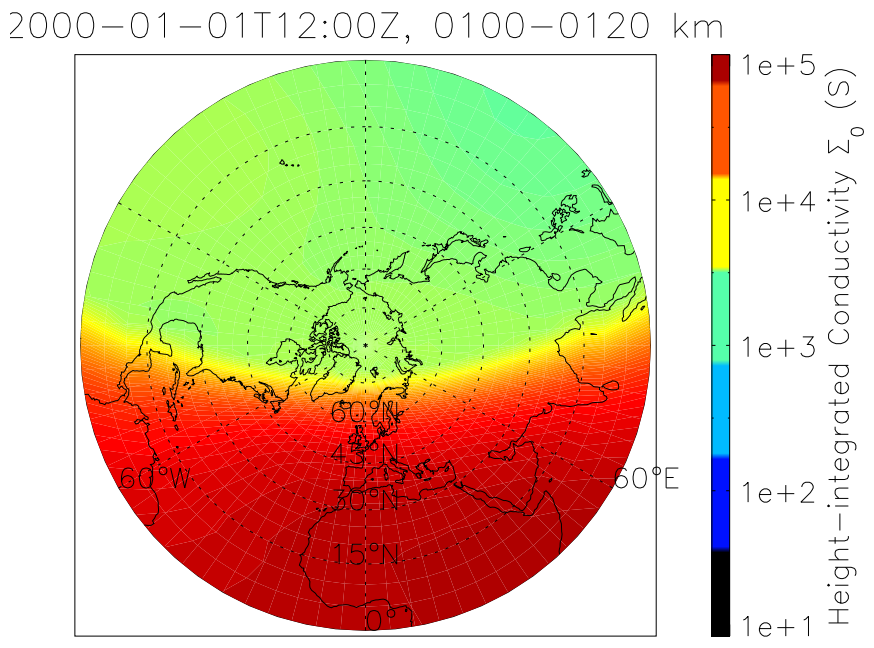

Figure 8: Stereo plot of height-integrated conductivity $\Sigma_{0}$ calculated by using the equations in Section 2.1 in the altitude range from 100 to $120 \mathrm{~km}$ at 12:00 UT on January 1, 2000.

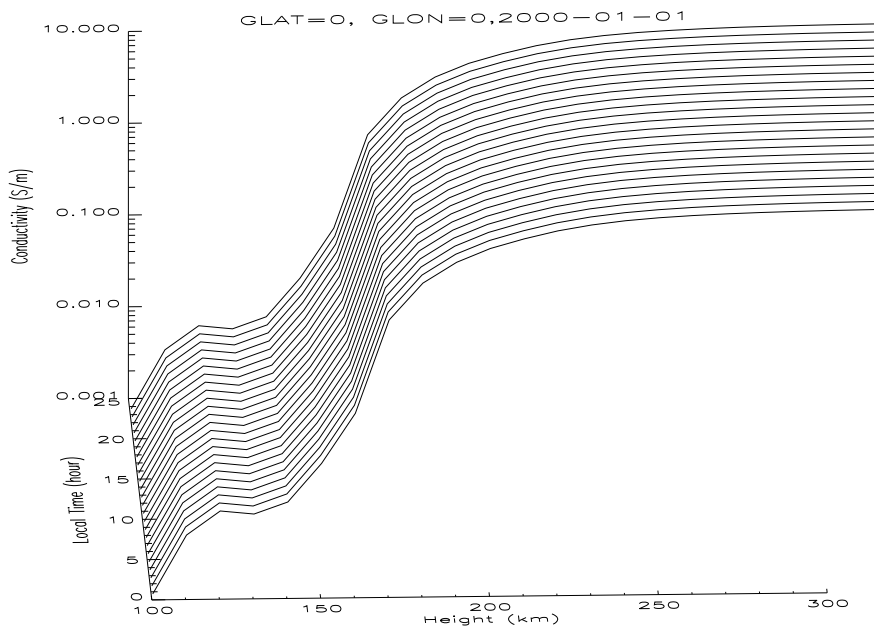

Figure 9: Time dependency of the height profile of the ionospheric conductivity $\sigma_{0}$ calculated by using the equations in Section 2.1 in the altitude range from 100 to $300 \mathrm{~km}$. 


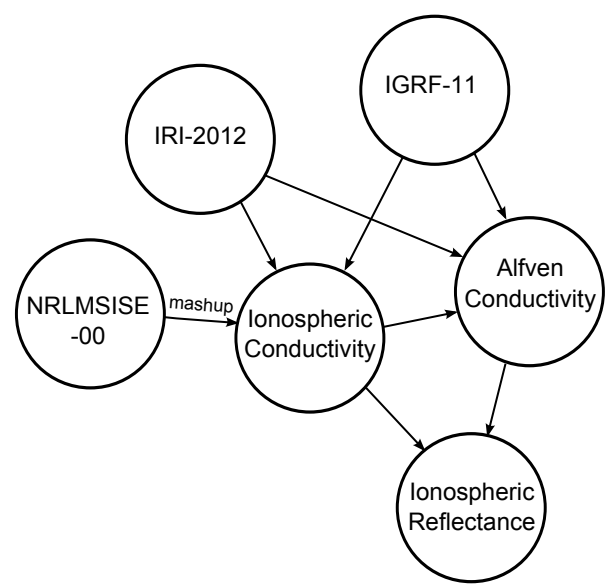

Figure 10: Dependency diagram of the models. The ionospheric conductivity model was created from NRLMSISE-00, IRI-2012, and IGRF-11 models. The IRI-2012 and IGRF-11 models can be reused by creating the Alfvén conductivity model. The Pedersen conductivity obtained from the ionospheric conductivity model and the Alfvén conductivity are also reused for derivation of the ionospheric reflectance of Alfvén waves. 